\title{
Identification of the Low\#Mass X\#Ray Binary and Faint X\#Ray Sources in NGC 6652
}

\section{Citation}

Heinke, C. O., P. D. Edmonds, and J. E. Grindlay. 2001. “Identification of the Low\#Mass X\# Ray Binary and Faint X\#Ray Sources in NGC 6652." The Astrophysical Journal 562 (1): 363-67. https://doi.org/10.1086/323493.

\section{Permanent link}

http://nrs.harvard.edu/urn-3:HUL.InstRepos:41399869

\section{Terms of Use}

This article was downloaded from Harvard University's DASH repository, and is made available under the terms and conditions applicable to Other Posted Material, as set forth at http:// nrs.harvard.edu/urn-3:HUL.InstRepos:dash.current.terms-of-use\#LAA

\section{Share Your Story}

The Harvard community has made this article openly available.

Please share how this access benefits you. Submit a story.

Accessibility 
ThE Astrophysical JouRnAL, 562:363-367, 2001 November 20

(C) 2001. The American Astronomical Society. All rights reserved. Printed in U.S.A.

\title{
IDENTIFICATION OF THE LOW-MASS X-RAY BINARY AND FAINT X-RAY SOURCES IN NGC 6652
}

\author{
C. O. Heinke, P. D. Edmonds, and J. E. Grindlay \\ Harvard-Smithsonian Center for Astrophysics, 60 Garden Street, Cambridge, MA 02138; \\ cheinke@cfa.harvard.edu, pedmonds@cfa.harvard.edu, josh@cfa.harvard.edu \\ Received 2001 May 30; accepted 2001 July 25
}

\begin{abstract}
We have detected three new X-ray point sources, in addition to the known low-mass X-ray binary (LMXB) X1832-330, in the globular cluster NGC 6652 with a Chandra 1.6 ks HRC-I exposure. Star 49 $\left(M_{V} \sim 4.7\right)$, suggested by Deutsch and coworkers as the optical candidate for the LMXB, is identified $\left(<0^{\prime \prime} 3\right)$ not with the LMXB, but with another, newly detected source (B). Using archival Hubble Space Telescope $(H S T)$ images, we identify $(<0$ ".3) the LMXB (A) and one of the remaining new sources (C) with blue variable optical counterparts at $M_{V} \sim 3.7$ and 5.3, respectively. The other new source (D) remains unidentified in the crowded cluster core. In the $0.5-2.5 \mathrm{keV}$ range, assuming a $5 \mathrm{keV}$ thermal bremsstrahlung spectrum and $N_{\mathrm{H}}=5.5 \times 10^{20}$, source A has intrinsic luminosity $L_{\mathrm{X}} \sim 5.3 \times 10^{35}$ ergs $\mathrm{s}^{-1}$. Assuming a $1 \mathrm{keV}$ thermal bremsstrahlung spectrum, B has $L_{\mathrm{X}} \sim 4.1 \times 10^{33} \mathrm{ergs} \mathrm{s}^{-1}$, while $\mathrm{C}$ and D have $L_{\mathrm{X}} \sim 8 \times 10^{32} \mathrm{ergs} \mathrm{s}^{-1}$. Source B is probably a quiescent LMXB, while source C may be either a luminous cataclysmic variable or a quiescent LMXB.

Subject headings: binaries: close — binaries: eclipsing — globular clusters: individual (NGC 6652) novae, cataclysmic variables - stars: neutron - stellar dynamics - X-rays: binaries
\end{abstract}

\section{INTRODUCTION}

Globular clusters have proven to be an excellent place to study stellar populations, dynamic evolution, and binary systems. Their high stellar densities allow their populations of white dwarfs and neutron stars to interact with cluster primordial binaries by exchange collisions (Hut et al. 1992). Stellar evolution and dynamic hardening of compact binaries within globulars lead to mass transfer and accretion onto the compact object, which is visible especially in the X-ray regime. Thus, X-ray studies of globular clusters are particularly effective at studying both compact objects and compact binaries.

Successful searches for low-mass X-ray binary (LMXB) optical counterparts have been undertaken for several clusters (Deutsch, Anderson, \& Margon 1998a and references therein; Homer et al. 2001). However, of the 12 globular cluster LMXBs in the Galaxy (see Grindlay 1993), at least six remain optically unidentified - those in Terzan 1, 2, 5, and 6, Liller 1, and NGC 6652. Of the separate population of low-luminosity X-ray sources (Hertz \& Grindlay 1983), many sources have now been positively identified with cataclysmic variables (CVs), as in NGC 6397 (Edmonds et al. 1999 and references therein), and with CVs, millisecond pulsars, and BY Draconis binaries in 47 Tucanae (Grindlay et al. 2001 and references therein).

The globular cluster NGC 6652 includes one previously known LMXB, X1832-330. This cluster is at Galactocentric radius $2 \mathrm{kpc}$ and $9.0 \pm 0.4 \mathrm{kpc}$ from the Sun and is $11.7 \pm 1.6$ Gyr old (Chaboyer, Sarajedini, \& Armandroff 2000). The LMXB was probably detected by $H E A O 1$ at a low level (Hertz \& Wood 1985). It was securely detected by ROSAT during the all-sky survey (Predehl et al. 1991), again during pointed ROSAT observations (Johnston, Verbunt, \& Hasinger 1996), and in type 1 X-ray bursts by both $A S C A$ and BeppoSAX (in't Zand et al. 1998; Mukai \& Smale 2000). A search for the optical counterpart of the LMXB was undertaken by Deutsch, Margon, \& Anderson (1998b; hereafter DMA98), unfortunately not including the entire ROSAT error circle for the LMXB. They were able to identify a plausible candidate (star 49); however, it is located $2.3 \sigma$ from the ROSAT position and is identified here with a lower luminosity source. This paper presents observations with Chandra as part of a campaign to measure precise positions for the remaining unidentified LMXBs. We also use Hubble Space Telescope (HST) archival images to identify optical counterparts.

\section{OBSERVATIONS AND OPTICAL IDENTIFICATIONS}

We observed NGC 6652 on 2000 May 23 with the High Resolution Camera-Imager (HRC-I) camera (Murray et al. $2000)$ on the Chandra $X$-Ray Observatory $(C X O)$. The Chandra mirrors give well-defined point-spread functions of half-power diameter 0"76 (Jerius et al. 2000), oversampled by the HRC-I, allowing relative positional accuracy to less than 0 ".3. The aspect reconstruction systems allow absolute positional accuracy of 0"6 $(1 \sigma$; Aldcroft et al. 2000).

Data were analyzed using the Chandra X-Ray Center CIAO software ${ }^{1}$ and standard processing, producing $1679 \mathrm{~s}$ of good data. The CXC source detection routine WAVDETECT (Dobrzycki et al. 1999) was used to detect sources in the exposure-corrected central field ( $4^{\prime}$ radius circle). The PROS variability test "vartst" was applied to each source.

Most of the HST data analyzed here (except for brief references to $B, V$, and $U$ data) are archival data from the 1997 September WFPC2 observations of B. Chaboyer (GO6517). Under this program, an orbit of short (23 s) F555W and $(20 \mathrm{~s}) \mathrm{F} 814 \mathrm{~W}$ images were obtained at two different pointings, followed by an orbit of 12 long (160 s) F555W images and an orbit of 12 long (160 s) F814W images. The second and third orbits were obtained at a fixed pointing with the center of NGC 6652 placed in the center of the PC. The 12 deep F555W and F814W images were cleaned of cosmic rays and combined into single images. Aperture photometry and point-spread function (PSF) fitting were used to derive instrumental $m_{555}$ and $m_{814}$ magnitudes,

\footnotetext{
${ }^{1}$ All CXC software is available at http://asc.harvard.edu/ciao.
} 


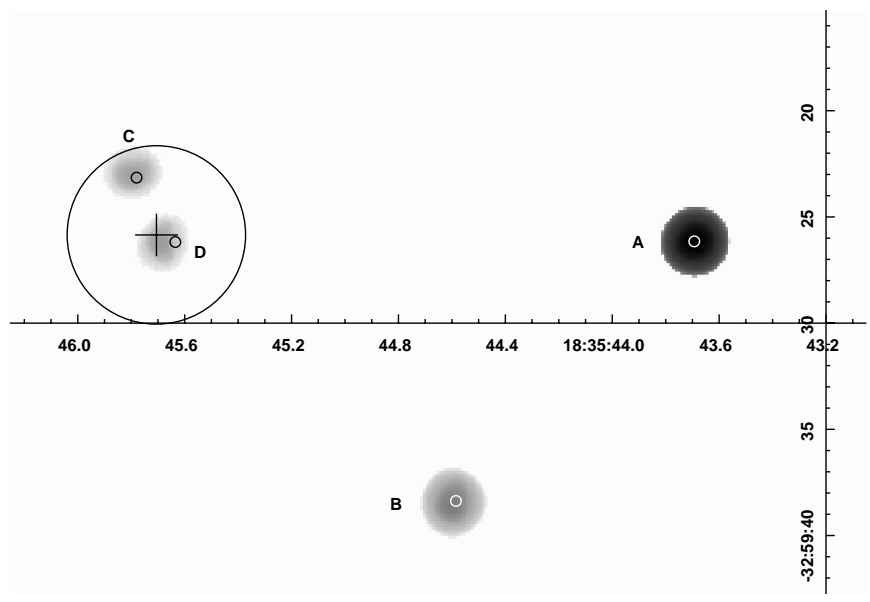

FIG. 1.-HRC-I Chandra image of NGC 6652. Image smoothed by wavelet detection algorithm WAVDETECT, part of Chandra analysis software package CIAO. The large circle represents one core radius (4..2; Harris 1996), and the cross marks the location and estimated uncertainty in the cluster center (J. E. Grindlay et al. 2001, in preparation). The small circles (radius 0 ".25, WF/PC2 FWHM for point sources) represent the locations (shifted to HRC frame) of the three likely optical counterparts, as well as the blue star near source D.

which were then converted into $V$ and $I$ using the prescription given in Holtzman et al. (1995). The precision of the PSF-fitting photometry along the main sequence was inferior to that obtained for aperture photometry, but was much less affected by crowding, especially near the cluster center. $H S T$ positional information makes reference to the Guide Star Catalog v.1.1, which has known absolute positional errors in the southern celestial hemisphere of $1^{\prime \prime}$ to 1.6 (Taff et al. 1990).

Six sources were detected in the central $4^{\prime}$ radius circle using WAVDETECT. Two sources more than $2^{\prime}$ from the core are probably not associated with the cluster, as the cluster half-mass radius is 0.65 , and the core radius is 0.07 (1999 June 22 version of the catalog described in Harris 1996). We were unable to find counterparts for these sources and ignore them in this paper. We detect four sources within $30^{\prime \prime}$ of the cluster center, including the bright LMXB X1832 - 330, hereafter source A (Fig. 1); the others we label "B," "C," and "D." All four sources are well detected: A has 9140 counts, B has 75 counts, C and D have 15 and 16 counts, respectively, with formal significances greater than $6 \sigma$ in each case. We consider all four of these sources to be associated with the cluster, since the probability that any of six sources within a $4^{\prime}$ circle would fall within $30^{\prime \prime}$ of the cluster center is $9 \%$. We give sources $\mathrm{A}, \mathrm{B}, \mathrm{C}$, and $\mathrm{D}$ the formal designations CXOGLB J183543.6-325926, CXOGLB J183544.5-325939, CXOGLB J183545.7325923, and CXOGLB J183545.6-325926, respectively, but we use the letter designations for this paper.

The second brightest source (source B) is detected in the HRC-I data at a position 0 ".8 from the position of star 49 (in the HST Guide Star Catalog frame). Star 49 was suggested by DMA98 as the counterpart for the LMXB, which we detect $17^{\prime \prime}$ away. Star 49 has measured $(U-B)=-0.9$ (DMA98) and shows variability of $1 \mathrm{mag}$, possibly with a 43.6 minute periodic modulation (Deutsch, Anderson, \& Margon 2000). DMA98 searched a $35^{\prime \prime} \times 35^{\prime \prime} H S T$ WF/PC1 field (not including source A; the GO-6517 data was not public then), for $U$-bright stars. They found only one object, star 49 , with $U-B$ color and magnitude similar to known LMXBs. Adding the GSC 1".6 (maximum) and HRC 0".6 absolute positional errors in quadrature gives a 1.7 $1 \sigma$ error circle. The 1.7 error circle is not crowded (being $\sim 4$ core radii from the center), so we confidently identify star 49 with source B.

By matching the GO-6517 and HRC coordinates for star 49 and source B, we performed a shift of the HRC coordinate frame by -0.04263 in R.A. and -0 ".609 in decl. The $H S T$ images then allowed the identification of $V-I$ excess optical counterparts for sources A and C within 0".01 and 0 "'3 of the corrected HRC positions. The derived X-ray and optical parameters of sources A through D are given in Table 1.

Figure 2 shows the $V$ versus $V-I$ color-magnitude diagrams (CMDs) resulting from PSF-fitting to both HST PC and WF data. The likely optical counterparts for sources A, $\mathrm{B}$, and $\mathrm{C}$ are shown, as are some other stars (circles, all are distant from X-ray positions) confirmed to be blue objects by visual examination of the $V$ and $I$ images.

Figure 3 shows the combined $V$ and $I$ time series for sources $A$ and $C$ (the time series for source $B$ is reported in DMA98). A zero-point correction was applied to the $I$ data so that $\langle V\rangle-\langle I\rangle=0$, and the errors were calculated from the median time series rms for stars within $0.25 \mathrm{mag}$ (typically $150-400$ stars) of either source A or C. Best-fitting

TABLE 1

X-Ray Source Parameters

\begin{tabular}{|c|c|c|c|c|c|c|c|}
\hline Src. & R.A. $^{\mathrm{a}}$ & Decl. & $F_{\mathrm{X}}^{\mathrm{b}}$ & $F_{\mathrm{X}} / F_{\mathrm{Opt}}^{\mathrm{c}}$ & $L_{\mathbf{X}}{ }^{\mathrm{d}}$ & $M_{V}$ & $V-I$ \\
\hline A. & $183543.649 \pm 0.001$ & $-325926.77 \pm 0.01$ & $5.9 \times 10^{-11}$ & 16 & $1.1 \times 10^{36}$ & 3.7 & 0.34 \\
\hline $\mathrm{B} \ldots \ldots$. & $183544.543 \pm 0.009$ & $-325938.97 \pm 0.09$ & $3.7 \times 10^{-13}$ & 0.3 & $4.1 \times 10^{33}$ & 4.7 & 0.85 \\
\hline $\mathrm{C} \ldots \ldots$ & $183545.751 \pm 0.011$ & $-325923.51 \pm 0.06$ & $7.3 \times 10^{-14}$ & 0.1 & $8 \times 10^{32}$ & 5.3 & -0.06 \\
\hline $\mathrm{D} \ldots \ldots$ & $183545.643 \pm 0.012$ & $-325926.74 \pm 0.15$ & $7.3 \times 10^{-14}$ & $\ldots$ & $8 \times 10^{32}$ & $\ldots$ & $\ldots$ \\
\hline
\end{tabular}

NotE.-Units of right ascension are hours, minutes, and seconds, and units of declination are degrees, arcminutes, and arcseconds.

a The errors are statistical centroiding errors; systematic errors on the absolute coordinates are 0".6 $(1 \sigma)$, from Aldcroft et al. 2000. Coordinates are shifted onto the HST Guide Star Catalog frame as described in the text.

${ }^{b}$ For $0.5-2.5 \mathrm{keV}$ band, received flux in ergs s${ }^{-1} \mathrm{~cm}^{-2}$, assuming $1 \mathrm{keV}$ thermal bremsstrahlung spectrum for sources $\mathrm{B}, \mathrm{C}$, and D, power law of $\alpha=1.86$ for A, $N_{\mathrm{H}}=5.5 \times 10^{20}$ for B, C, and D and $N_{\mathrm{H}}=4 \times 10^{21}$ for A.

${ }^{\mathrm{c}}$ Ratio of X-ray (0.5-2.5 keV band) to optical/UV flux (using $\log F_{\text {Opt }}=-0.4 V-3.96$, from Verbunt et al. 1997).

${ }^{\mathrm{d}}$ Intrinsic luminosity in ergs s ${ }^{-1}, 0.5-2.5 \mathrm{keV}$, assuming $N_{\mathrm{H}}$ and spectra as above, $(m-M)_{V}=15.15, E(B-V)=0.12$, $d=9.0 \mathrm{kpc}$, from Chaboyer et al. 2000 . 


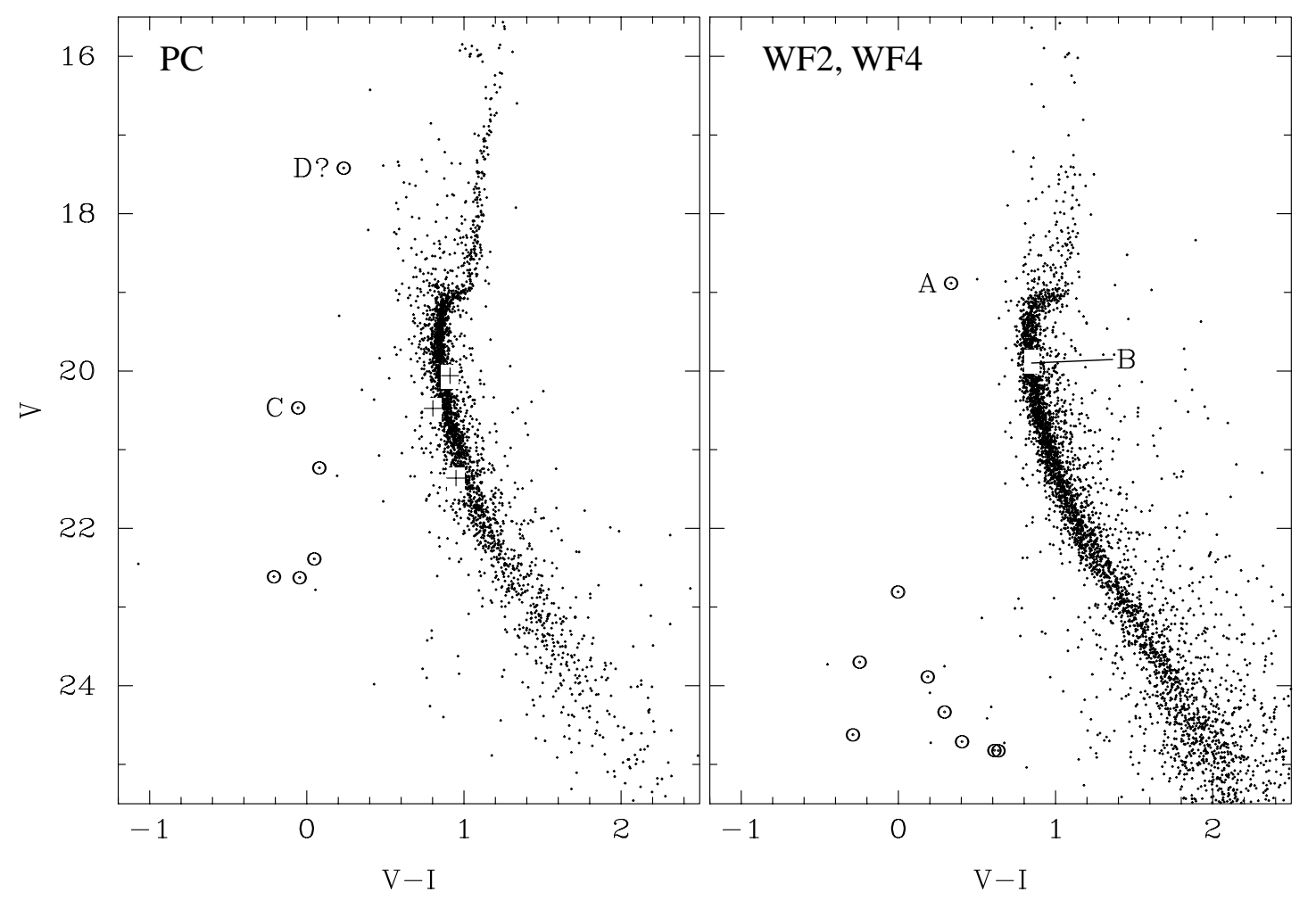

FIG. 2.- $V-I$ color-magnitude diagrams of NGC 6652, showing three probable counterparts (A, B, C) of detected X-ray sources. Left: The CMD for the PC. Right: The CMD for WF2 and WF4 combined (note that undersampling and saturation affect the giant branch in the WF CMD). Note that source B falls on the main sequence in $V-I$. A bright blue nonvariable star near the location of source D is indicated as "D?". Crosses mark the location of three normal stars also falling within the $3 \sigma$ error circle for C. Several blue stars are also indicated by circles, see text.

sinusoids have been overplotted showing possible periodicities for $\mathrm{A}$ and $\mathrm{C}$.

\section{RESULTS}

\subsection{Source A: $L M X B$}

The blue counterpart for the LMXB has $V=18.9$, $V-I=0.4$; this is $0.4 \mathrm{mag}$ above the turnoff and $0.4 \mathrm{mag}$ blueward of the main sequence in $V-I$, similar to other known LMXBs. Our suggested counterpart of source A is partly cut off in archival HST $U$ images, possibly explaining why it was not identified in DMA98. However, the peak pixel value at the edge of the chip clearly shows that this star is UV bright.

A time series analysis of the $V$ and $I$ exposures shows a clear $0.08 \mathrm{mag}$ variability, with $\mathrm{rms}$ variation 4.4 and $6.2 \sigma$ in $V$ and $I$, respectively, where $\sigma$ is defined as the median rms variation of stars within 0.25 mag of the star. Possible periods of 0.92 , or $2.22 \mathrm{hr}$ (or $4.44 \mathrm{hr}$ for ellipsoidal variations), as well as some nonperiodic flickering, are observed (see Fig. 3). The false alarm probability (FAP; see Scargle 1982) is $\sim 0.3 \times 10^{-3}$ for each period individually, although the short temporal coverage does not allow us to distinguish between these periods. We suggest one of these is the orbital period of the system, leading to an orbital separation of (for $M_{\text {total }}=1.6 M_{\odot}$ ) either $3.9 \times 10^{10} \mathrm{~cm}$, $7.0 \times 10^{10} \mathrm{~cm}$, or $1.1 \times 10^{11} \mathrm{~cm}$, respectively (Frank, King, \& Raine 1992). Either a low-mass M dwarf or a white dwarf would be a plausible secondary, depending on which period is the real one.

The absolute magnitude $M_{V}=3.7$ of the optical counterpart is comparable to those of ultracompact $(P<1 \mathrm{hr})$
LMXBs in globular clusters: 3.7 for X1820-303 in NGC 6624, and 5.6 for X1850 - 087 in NGC 6712 (van Paradijs \& McClintock 1994). X-ray reprocessing into optical light in accretion disks tends to follow the X-ray luminosity to absolute magnitude scaling relation proposed by van Paradijs \& McClintock (1994, Fig. 2), $M_{V}=1.57( \pm 0.24)$ $-2.27( \pm 0.32) \log \Sigma$, where $\Sigma$ is $\left(L_{\mathrm{X}} / L_{\mathrm{Edd}}\right)^{1 / 2}(P / 1 \mathrm{hr})^{2 / 3}$. For $M_{V}=3.7$, we find $\log \Sigma=-1$ to -0.5 for periods from 0.92 to $4.4 \mathrm{hr}$. All three proposed periods place this LMXB within the scatter of known LMXBs.

The HRC-I has no spectral resolution, yet our flux determination depends upon the assumed spectrum. By using a partial covering absorber $\left(2 / 3\right.$ covered by $\left.8 \times 10^{21} \mathrm{~cm}^{-2}\right)$ and a power law with photon index 1.86, Mukai \& Smale (2000) fitted both their ASCA GIS spectrum and the second ROSAT PSPC spectrum. From their recorded flux we derive $L_{\mathrm{X}}(2-8 \mathrm{keV})=1.5 \times 10^{36}$ in the $A S C A$ range or $L_{\mathrm{X}}(0.5-2.5 \mathrm{keV})=6.8 \times 10^{35} \mathrm{ergs} \mathrm{s}^{-1}$ extrapolated to the ROSAT range. The fitted partial covering absorber suggests variability in the $N_{\mathrm{H}}$ column, which would also explain the differences in spectral fitting by ROSAT data (Johnston et al. 1996; Verbunt et al. 1995). BeppoSAX observations (in't Zand et al. 1998) revealed type I bursts (observed also by Mukai \& Smale 2000) and persistent emission at $L_{\mathbf{X}}(2-8$ $\mathrm{keV}$ ) $=5.2 \pm 1.0 \times 10^{35} \mathrm{ergs} \mathrm{s}^{-1}$ (adjusted for the change in distance to $9.0 \mathrm{kpc}$, without absorption). The low-level emission they observed agrees with both ROSAT observations of low-level luminosity extended to the harder band, for a photon index of 1.86 .

We use a power-law spectrum with photon index 1.86 and a range of $N_{\mathrm{H}}$ between $5.5 \times 10^{20}$ and $4 \times 10^{21}$, the first being the average cluster value and the second the 


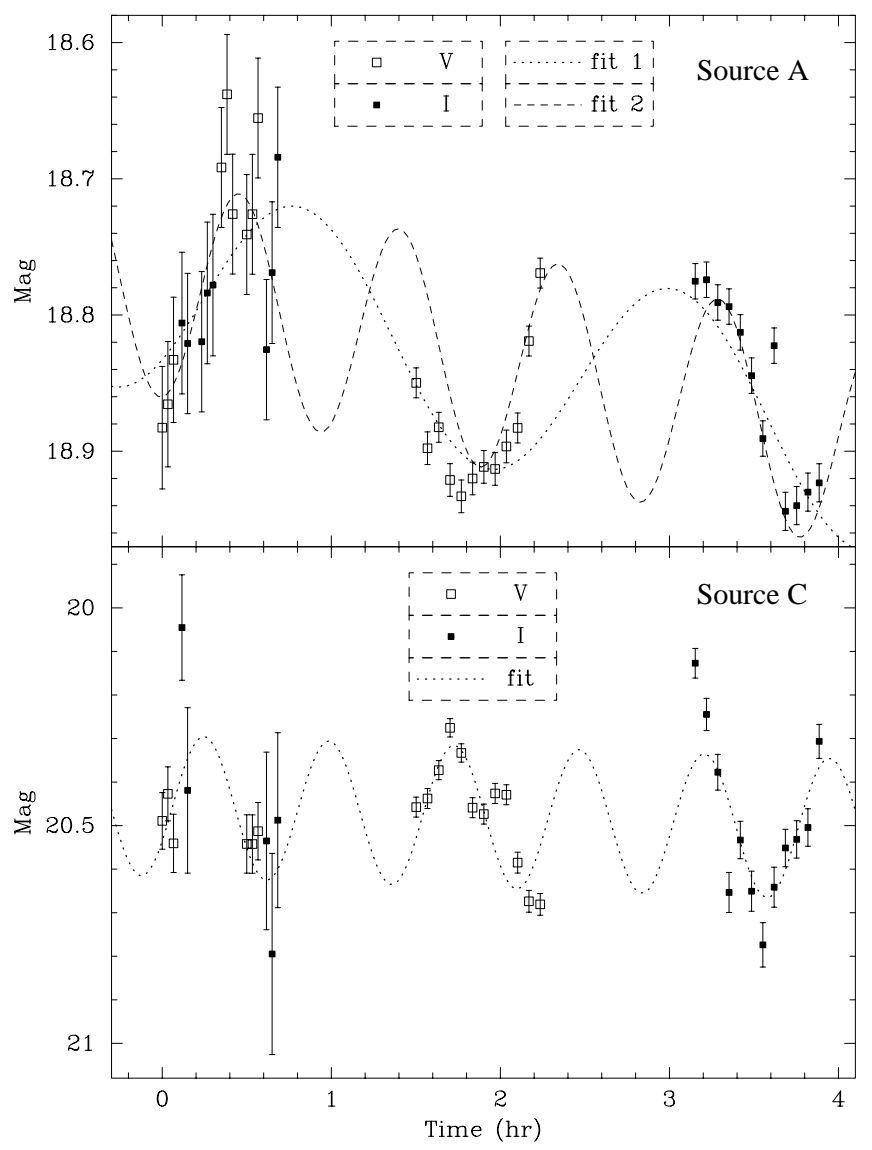

Fig. 3.-Optical light curves for sources A and C from three HST orbits. The open squares represent $V$ data and the filled squares represent $I$ data, shifted to correct for time-averaged color. Two sinusoidal fits are plotted for source A, with periods of 0.92 (fit 2) and 2.22 (fit 1) hr.

maximum value for A from $A S C A$ observations. These give intrinsic luminosity ranges of $L_{\mathrm{X}}(0.5-2.5 \mathrm{keV}) \sim 5.1 \times 10^{35}$ to $1.1 \times 10^{36} \mathrm{ergs} \mathrm{s}^{-1}$, similar to the ROSAT observations in 1990 and 1992 (Verbunt et al. 1995; Johnston et al. 1996), and $L_{\mathrm{X}}(2-8 \mathrm{keV}) \sim 5.3 \times 10^{35}$ to $1.2 \times 10^{36} \mathrm{ergs} \mathrm{s}^{-1}$, comparable to the ASCA and BeppoSAX observations in 19961997 (Mukai \& Smale 2000; in't Zand et al. 1998). We see no significant variability during the $1.6 \mathrm{ks}$ observation. Mukai \& Smale (2000) note that no eclipses have been seen and that we directly observe the neutron star during the type I bursts, ruling out a high-inclination geometry. This suggests that the optical variability may be due to orbital variations of the heated face of the secondary.

\subsection{Source B: $q L M X B$}

The X-ray luminosity of source $\mathrm{B}, \quad L_{\mathrm{X}}(0.5-2.5$ $\mathrm{keV})=4.1 \pm 0.5 \times 10^{33}$ for a $1 \mathrm{keV}$ bremsstrahlung spectrum, suggests a quiescent LMXB since CVs generally do not reach this luminosity (Verbunt et al. 1997). Source B is the only source to show clear X-ray variability, at the $95 \%$ level in the Cramer-Von Mises test (Daniel 1990). It is possible that this source is responsible for the type I bursts seen with $A S C A$ and BeppoSAX (in't Zand et al. 1998; Mukai \& Smale 2000), since neither telescope could resolve source B from the bright LMXB, source A, only 17" distant.

DMA98 identified star 49 as the bright LMXB counterpart based on $U-B$ excess $(U-B=-0.9,1.2$ mag blue- ward of the main sequence [MS]) and later (in Deutsch et al. 2000) added weight to the identification by discovering $\sim 1 \mathrm{mag}$ flickering and a possible period of 43.6 minutes. We confirm the variability, at $24 \sigma(V)$ and $21 \sigma(I)$, and identify the same possible optical period, but with an FAP of 0.027 , the least significant FAP of the power spectrum peaks for the three optical counterparts.

If the 43.6 minute period is the real binary period, a main sequence companion is ruled out since the implied orbital separation for a $2 M_{\odot}$ system is $3.6 \times 10^{10} \mathrm{~cm}$, less than the radius of even an M2 star, implying a double-degenerate system. However, this identification suffers a problem in that star 49 falls onto the MS in $V-I$ (see Fig. 2, Table 1). The system also lies on the MS in an instrumental $V$ versus $B-V$ CMD from further archival analysis. The MS colors suggest that light from a main-sequence secondary is dominant in $V$ and $I$ and that the weak accretion disk provides the $U$ excess (see DMA98). However, this interpretation cannot explain why both $V$ and $I$ vary in tandem by $1 \mathrm{mag}$ (Deutsch et al. 2000, Fig. 1) without invoking eclipses, nor does it explain the large flickering (which generally implies a bright, blue disk).

We considered the possibility that the identification suffers source confusion (background or foreground stars contributing $V, I$ light), but there is no evidence for blending in the HST image. We consider it most likely that the 43.6 minute period is spurious, but the 1 mag variability remains a mystery.

\subsection{Source $C$ : $C V$ or $q L M X B$ ?}

The suggested counterpart of source $\mathrm{C}$ lies only $3^{\prime \prime}$ from the cluster center, $\alpha=18^{h} 35^{m} 45^{\mathrm{s}} 66, \quad \delta=-32^{\circ} 59^{\prime} 26^{\prime \prime} .5$ (J2000), from J. E. Grindlay et al. (2001, in preparation). This is within one core radius (4".2, Harris 1996). Its $V-I$ color, $M_{V}$, and $F_{\mathrm{X}} / F_{V}$ (see Table 1, Fig. 2) suggest a $C V$, since light from field CVs is usually dominated by the hot accretion disk throughout the optical range. Although both the X-ray luminosity and $M_{V}$ are bright for a CV, bright magnetic CVs (IPs; see Barrett, Singh, \& Mitchell 1999), or CVs in outburst, reach this range. Archival GO-6095 HST WFPC2 data shows $\mathrm{C}$ at similar brightness and color, and it is unlikely that we have caught $\mathrm{C}$ in outburst twice. A quiescent LMXB is the other plausible interpretation. However, the accretion disk in a low- $\dot{m}$ system is expected to be truncated due to a mass transfer instability leading to an ADAF flow, and the light from the secondary should be dominant for $V-I$.

The optical time series for source $\mathrm{C}$ shows clear variability (see Fig. 3), at the $5.0 \sigma(V)$ and $7.6 \sigma(I)$ levels. We find a possible periodicity of 44.4 minutes (close to the 48 minute $H S T$ half-period), semi-amplitude of $0.16 \mathrm{mag}$, and FAP of $0.88 \times 10^{-2}(\sim 1 \%)$. Three other stars within a $3 \sigma$ error circle (indicated by plus signs in Fig. 2) show no variability at the $2 \sigma$ level. The time series for $\mathrm{C}$ shows significant flickering, which might combine with the periodic $H S T$ window to generate the possible 44.4 minute periodicity. This period is disturbingly close to the suggested 43.6 minute period for source $\mathrm{B}$, indicating that both periods may be spurious.

\subsection{Source D: Multiple Sources?}

The final source, D, lies less than 0.4 from the cluster core (as given above). The $\mathrm{X}$-ray flux shows no significant variability. A bright blue star $(V=17.4, V-I=0.2)$ is located 
0"7 away from the corrected X-ray position (see Fig. 1) but shows no variability (significance $\lesssim 2 \sigma$ ). Based on its nonvariability and magnitude (see Fig. 2), we judge it to be a blue straggler and unlikely to be the counterpart. Accurate photometry of source D's error circle down to the expected magnitude of CVs or qLMXBs $\left(m_{V} \gtrsim 20\right)$ may be impossible due to the overlapping PSFs from nearby blue stragglers and red giants.

It is quite possible that source $\mathrm{D}$ is really multiple sources overlapping within the central core, where the concentration of close binaries and thus X-ray sources is expected to be high. Possibilities for source D include a qLMXB, a bright millisecond pulsar, and/or one or more CVs.

\section{DISCUSSION}

Our detection limit in this observation was $L_{\mathbf{X}}(0.5-2.5$ $\mathrm{keV}) \sim 4 \times 10^{32} \mathrm{ergs} \mathrm{s}^{-1}$, for a $90 \%$ confidence detection threshold 4 count source, background being 0.04 counts $\operatorname{arcsec}^{-2}$ at the core. Most detected field CVs are at $\lesssim 10^{32}$ ergs $\mathrm{s}^{-1}$ in this band, and would thus be missed by this short exposure. The total detected emission from within 1 core radius, assuming a $1 \mathrm{keV}$ thermal bremsstrahlung spectrum, was $1.5 \times 10^{33} \mathrm{ergs} \mathrm{s}^{-1}$ and can be accounted for by the two detected sources. A deeper ACIS exposure could measure spectra, confirm the possible 43.6 minute period for source B, and identify low-luminosity sources such as CVs. Several potential CVs or hot white dwarfs on the WF2 and WF4 chips are indicated in Figure 2. Additional blue nonvariable stars are noted on the PC chip; their lack of variability and blue colors resemble the nonflickerers noted in NGC 6397 and proposed to be $\mathrm{He}$ white dwarfs (Edmonds et al. 1999). However, these could be field stars. Using the figures from Ratnatunga \& Bahcall (1985) for Galactic star counts in the direction of NGC 6652, we estimate that we should see $\sim 1.2$ stars with $B-V<0.8$ between $19<m_{V}<23$ in the PC field, and $\sim 11$ stars with $B-V<0.8$ between $21<m_{V}<25$ in the combined WF2 and WF4 fields. Thus, the WF2 and WF4 blue stars could be explained by field contamination, but it is unlikely that all the blue stars in the PC are field stars.

The locations of the sources in this cluster are intriguing. Sources $\mathrm{C}$ and $\mathrm{D}$ are within one core radius of the center, but $\mathrm{A}$ and $\mathrm{B}$ (each expected to be $\sim 1.6-2 M_{\odot}$ ) are at least 6 and 4 core radii, respectively, from the center of the cluster. They are not in dynamic equilibrium in the cluster, since binaries tend to sink toward the cluster core within the local relaxation time (Hut et al. 1992). The half-mass relaxation time for NGC 6652 is $3.5 \times 10^{8} \mathrm{yr}$, and both sources are well within the half-mass radius of $39^{\prime \prime}$ (Harris 1996). In a separate paper, we consider the possibility that they have been ejected from the cluster core by examining the radial distribution of all 12 bright LMXBs using both our new Chandra positions and updated cluster parameters (centers and core radii; J. E. Grindlay et al. 2001, in preparation).

This work was supported in part by Chandra grant GO01034A. The ability to make this observation is a testament to the dedication and hard work of the Chandra team.

\section{REFERENCES}

Aldcroft, T. L., Karovska, M., Cresitello-Ditmar, M. L., Cameron, R. A., \& Markevitch, M. L. 2000, Proc. SPIE, 4012, 650

Barrett, P., Singh, K. P., \& Mitchell, S. 1999, in ASP Conf. Ser. 157, Annapolis Workshop on Magnetic Cataclysmic Variables, C. Hellier \& K. Mukai (San Francisco: ASP), 180

Chaboyer, B., Sarajedini, A., \& Armandroff, T. E. 2000, ApJ, 120, 3102

Daniel, W. W. 1990, Applied Nonparametric Statistics (2d ed.; Boston: PWS-Kent)

Deutsch, E. W., Anderson, S. F., \& Margon, B. 1998a, ApJ, 493, 775

.2000, ApJ, 530, L21

Deutsch, E. W., Margon, B., \& Anderson, S. F. 1998b, AJ, 116, 1301 (DMA98)

Dobrzycki, A., Ebeling, H., Glotfelty, K., Freeman, P., Damiani, F., Elvis, M., \& Calderwood, T. 1999, CHANDRA Detect 1.1 User Guide (Cambridge: CXC)

Edmonds, P. D., Grindlay, J. E., Cool, A. M., Cohn, H., Lugger, P., \& Bailyn, C. 1999, ApJ, 516, 250

Frank, J., King, A., \& Raine, D. 1992, Accretion Power in Astrophysics (2d ed.; Cambridge: Cambridge Univ. Press)

Grindlay, J. E. 1993, in ASP Conf. Ser. 48, The Globular Clusters-Galaxy Connection, ed. S. R. Majewski (San Francisco: ASP), 156

Grindlay, J. E., Heinke, C. O., Edmonds, P. D., \& Murray, S. S. 2001, Science, 292, 2290

Harris, W. E. 1996, AJ, 112, 1487

Hertz, P. \& Grindlay, J. E. 1983, ApJ, 275, 105

Hertz, P. \& Wood, K. S. 1985, ApJ, 290, 171

Holtzman, J. A., Burrows, C. J., Casertano, S., Hester, J. J., Trauger, J. T., Watson, A. M., \& Worthey, G. 1995, PASP, 107, 1065

Homer, L., Anderson, S. F., Margon, B., Deutsch, E. W. \& Downes, R. A. 2001, ApJ, 550, L155

Hut, P., et al. 1992, PASP, 104, 981

in't Zand, J. J. M., Verbunt, F., Heise, J., Muller, J. M., Bazzano, A., Cocchi, M., Natalucci, L., \& Ubertini, P. 1998, A\&A, 329, L37

Jerius, D., Donnelly, R. H., Tibbetts, M. S., Edgar, R. J., Gaetz, T. J., Schwartz, D. A., van Speybroeck, L. P., \& Zhao, P. 2000, Proc. SPIE, 4012, 17

Johnston, H. M., Verbunt, F., \& Hasinger, G. 1996, A\&A, 309, 116

Mukai, K., \& Smale, A. P. 2000, ApJ, 533, 352

Murray, S. S., et al. 2000, Proc. SPIE, 4012, 68

Predehl, P., Hasinger, G., \& Verbunt, F. 1991, A\&A, 246, L21

Ratnatunga, K. U., \& Bahcall, J. N. 1985, ApJS, 59, 63

Scargle, J. D. 1982, ApJ, 263, 835

Taff, L. G., et al. 1990, ApJ, 353, L45

van Paradijs, J., \& McClintock, J. E. 1994, A\&A, 290, 133

Verbunt, F., Bunk, W., Hasinger, G., \& Johnston, H. M. 1995, A\&A, 300, 732

Verbunt, F., Bunk, W. H., Ritter, H., \& Pfefferman, E. 1997, A\&A, 327, 602 